\title{
Mucoepidermoid Carcinoma of the Parotid Gland in a Patient with Duchenne Muscular Dystrophy: A Case Report
}

\author{
Geun-Jeon Kim, MD ${ }^{1}$, Dong-Hyun Lee, MD ${ }^{1}$, Chul-Woo Yang, MD, PhD' and Dong-II Sun, MD, PhD ${ }^{1^{*}}$
}

${ }^{1}$ Department of Otolaryngology-Head and Neck Surgery, College of Medicine, The Catholic University of Korea, South Korea

${ }^{2}$ Department of Nephrology, College of Medicine, The Catholic University of Korea, South Korea

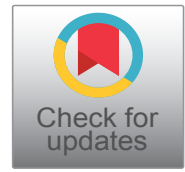

*Corresponding author: Dong-II Sun, MD, PhD, Department of Otolaryngology-Head and Neck Surgery, 505 Banpodong, Seochogu Kangnam St. Mary's Hospital, The Catholic University of Korea, Seoul, 137-040, South Korea, Tel: +82-2-22586212, Fax: +82-2-595-1354

\begin{abstract}
We report the co-occurrence of Duchenne muscular dystrophy (DMD) and mucoepidermoid carcinoma (MEC) of the parotid gland. A 33-year-old male presented with a parotid gland tumor of benign appearance. After surgery, the patient's serum creatine kinase level exceeded 20,000 IU/L (normal, 50-200 IU/L); his potassium level was also high $(7.2 \mathrm{mEq} / \mathrm{L})$, as were his aspartate aminotransferase, alanine aminotransferase, and lactate dehydrogenase levels. Genetic testing revealed the deletion of exons 10-29 on chromosome Xp21, and the patient was diagnosed with DMD. The resected specimen revealed evidence of lowgrade MEC. The patient underwent further treatment with radiotherapy.
\end{abstract}

\section{Keywords}

Mucoepidermoid cancer, Parotid gland cancer, Duchenne muscular dystrophy

\section{Introduction}

Duchenne muscular dystrophy (DMD), a rare, progressive myopathy, is a recessive disorder caused by mutations on the short arm of the $X$ chromosome. In most affected males, molecular deletions in the dystrophin gene disrupt dystrophin translation, leading to muscle cell membrane defects [1].

Salivary gland malignancies account for $<5 \%$ of all head and neck cancers, $70 \%$ of which are parotid gland malignancies [2]. Mucoepidermoid carcinoma (MEC) is the most common histological subtype, comprising $50 \%$ of all parotid malignancies [3].

Recent studies have shown an association between the DMD gene and neoplastic formation; however, this case report is the first to document co-occurrence of parotid gland cancer and DMD.

\section{Case Report}

A 33-year-old male presented to the Otolaryngology-Head and Neck Surgery Department with facial swelling in the right parotid area for the past 2 months. The patient presented only facial swelling with parotid mass, he didn't complain any general or neurologic problems.

Computed tomography and magnetic resonance imaging of the head and neck before surgery revealed a 3 $\times 3 \mathrm{~cm}$ mass confined to the deep lobe of the parotid gland with a benign appearance, suspected as a benign mixed tumor or Warthin tumor (Figure 1). Moreover, the sono-guided biopsy specimen was benign.

A slight elevation in the alanine aminotransferase (ALT) level (54 IU/L) was the only abnormal laboratory finding before surgery.

Surgical excision of the tumor was performed under general anesthesia. On examination, the tumor was solid with poorly defined margins and facial nerve involvement. Histological examination of the frozen biopsy specimen during surgery indicated that the tumor was

Citation: Geun-Jeon K, Dong-Hyun L, Chul-Woo Y, Dong-II S (2020) Mucoepidermoid Carcinoma of the Parotid Gland in a Patient with Duchenne Muscular Dystrophy: A Case Report. Int J Oncol Res 3:020. doi.org/10.23937/2643-4563/1710020

Accepted: January 20, 2020; Published: January 22, 2020

Copyright: (c) 2020 Geun-Jeon K, et al. This is an open-access article distributed under the terms of the Creative Commons Attribution License, which permits unrestricted use, distribution, and reproduction in any medium, provided the original author and source are credited. 


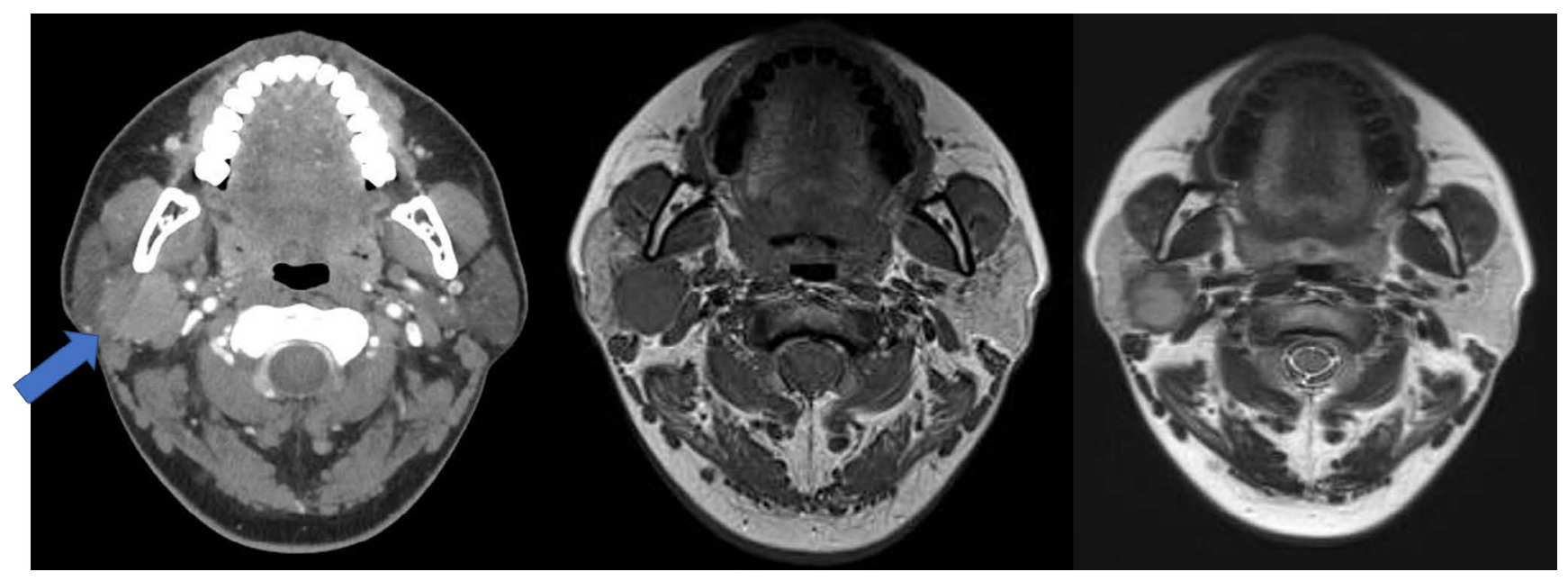

Figure 1: Radiological findings. A $27 \times 26 \times 39 \mathrm{~mm}$ mass was observed in the deep portion of the right parotid gland using a) Contrast-enhanced computed tomography; b) T1-weighted magnetic resonance imaging (MRI); c) T2-weighted MRI.

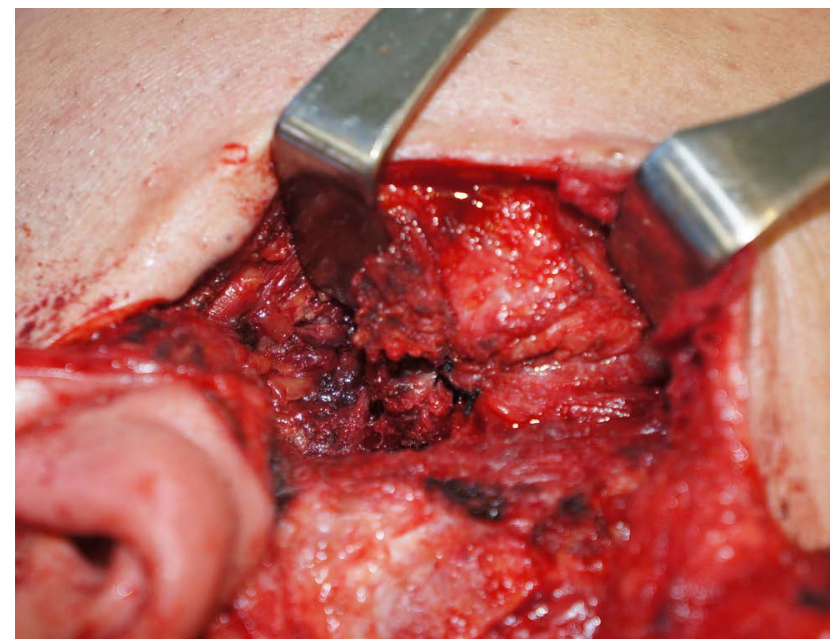

Figure 2: Surgical findings. The tumor was adhered to the facial nerve, from the trunk to the branches.

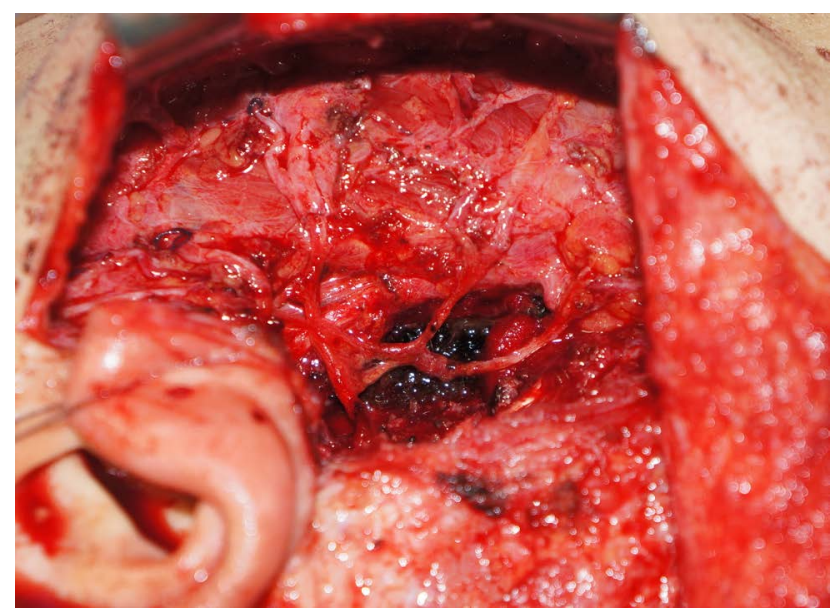

Figure 3: The resected facial nerve branches were reconstructed using cervical plexus grafts.

malignant; therefore, we performed a radical parotidectomy including the facial nerve. The tumor adhered tightly to the facial nerve, from the trunk at the stylomastoid foramen to the branches. The distal portion of

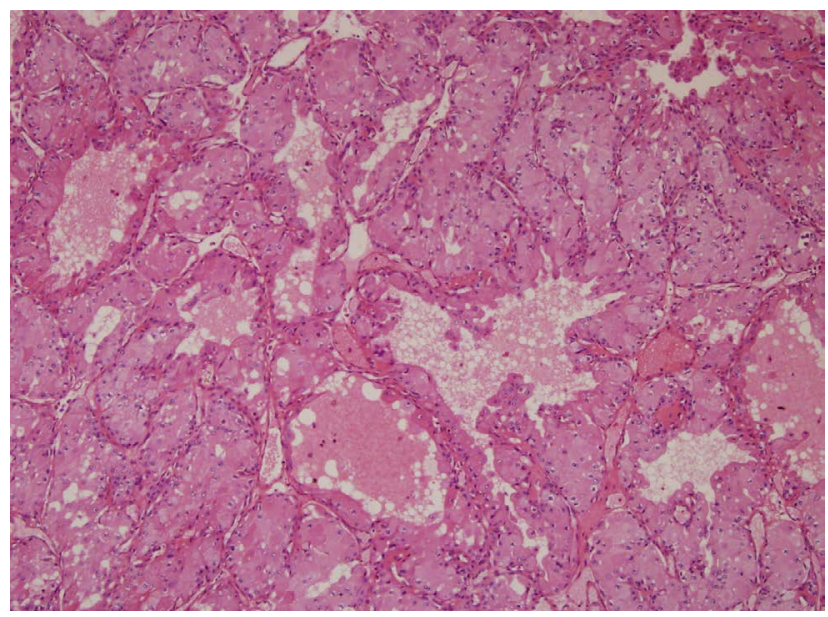

Figure 4: Histological examination revealed a low-grade mucoepidermoid carcinoma, with perineural invasion and negative margin status.

each branch was dissected and preserved, and the portion from the nerve trunk to the proximal part of the branches was sacrificed (Figure 2). The temporal, zygomatic, buccal, and marginal mandibular branches of the facial nerve were reconstructed from the nerve trunk using cervical plexus grafts (Figure 3 ).

On the day after surgery, the patient passed bloody urine. His serum creatine kinase (CK) level exceeded 20,000 IU/L (normal, 50-200 IU/L); his potassium level was also high $(7.2 \mathrm{mEq} / \mathrm{L})$, as were his aspartate aminotransferase (AST), ALT, and lactate dehydrogenase (LDH) levels.

Based on these findings, rhabdomyolysis was suspected. The anesthetics and neuromuscular blocking agents used, perioperative condition, and prolonged immobility were investigated as possible causes of the rhabdomyolysis; inflammatory myopathy and a metabolic disorder were also considered as possible etiologies. The patient underwent fluid resuscitation and urine alkalization to normalize his CK levels, and genetic 
testing was performed for further evaluation. Genetic testing of the dystrophin gene revealed the deletion of exons 10-29 on chromosome Xp21, characteristic of DMD, and the patient was diagnosed with DMD.

Histopathological examination of the resected specimen revealed evidence of low-grade MEC (Figure 4). The patient underwent additional treatment with radiotherapy. Five months after radiation therapy, CK (2703 IU/L), AST (72 IU/L), ALT (81 IU/L), and LDH (534 IU/L) levels remained high; however, kidney function recovered fully and further management was deemed unnecessary. The patient recovered well with no signs of recurrence.

\section{Discussion}

DMD, the most common fatal neuromuscular disease in children, is caused by a mutation in the dystrophin protein [4], has an incidence of one in 5000 boys and presents in early childhood with proximal muscle weakness [5]. DMD has not been shown to predispose individuals to cancer [6]. Genetic diseases are often predisposing factors for neoplastic processes because of alterations in oncogenes and tumor suppressor genes (cancer family syndromes) or, in a less overt way, by causing an imbalance in the cell cycle [7].

Since the first report of concomitant DMD and rhabdomyosarcoma, the association between the diseases has been investigated in children. Investigation of the abnormal molecular patterns seen in genetic diseases associated with cancer may shed light on the mechanisms underlying the malignant transformation of cells. DMD is not associated with immune system dysfunction or susceptibility to malignant disease in childhood or early adulthood. Because survival beyond the third decade of life is extremely rare in cases of DMD, the increased risk of cancer associated with older age is not a factor [8]. Previous reports showed a high frequency of $D M D$ intragenic deletions that were associated with the progression of myogenic tumors, assigning DMD a tumor suppressor activity in these types of cancer. However, there are only few reports that analyze DMD in non-myogenic tumors. Leonela, et al. [9] designed to examine DMD expression and genetic alterations in non-myogenic tumors using public repositories. These results support that DMD expression and genetic alterations are frequent and relevant in non-myogenic tumors. Our case report is the first to detail the occurrence of parotid gland cancer and DMD. Also, it is important to note that patients with DMD may develop acute rhabdomyolysis after surgery; volatile anesthetics have been associated with rhabdomyolysis in patients with DMD. In this case, the patients underwent gener- al anesthesia using sevoflurane as volatile anesthetics. Furthermore, several studies have shown that succinylcholine can trigger acute rhabdomyolysis in these patients [10] causing muscle necrosis, gross elevation of CK, and myoglobinuria; the resulting hyperkalemia, metabolic acidosis, and acute renal failure are predisposing factors for cardiac dysrhythmia. Therefore, patients with DMD should be monitored closely during surgery, regardless of the anesthetic used.

\section{Conclusion}

Although our findings do not indicate a relationship between DMD and parotid cancer, our case report is the first to document parotid cancer in a patient with DMD.

\section{Conflict of Interests}

The authors declare that there is no financial conflict of interests regarding the publication of this article.

\section{References}

1. Lovering RM, Porter NC, Bloch RJ (2005) The muscular dystrophies: From genes to therapies. Phys Ther 85: 13721388.

2. Ettl T, Schwarz-Furlan S, Gosau M, Reichert TE (2012) Salivary gland carcinomas. Oral Maxillofac Surg 16: 267-283.

3. Pinkston JA, Cole $P$ (1999) Incidence rates of salivary gland tumors: Results from a population-based study. Otolaryngol Head Neck Surg 120: 834-840.

4. Love DR, Davies KE (1989) Duchenne muscular dystrophy: The gene and the protein. Mol Biol Med 6: 7-17.

5. Moat SJ, Bradley DM, Salmon R, Clarke A, Hartley L (2013) Newborn bloodspot screening for Duchenne muscular dystrophy: 21 years experience in Wales (UK). Eur $\mathrm{J}$ Hum Genet 21: 1049-1053.

6. Pappo AS, Shapiro DN, Crist WM (1997) Rhabdomyosarcoma. Biology and treatment. Pediatr Clin North Am 44: 953-972.

7. Quesnel S, Malkin D (1997) Genetic predisposition to cancer and familial cancer syndromes. Pediatr Clin North Am 44: 791-808.

8. Rossbach HC, Lacson A, Grana NH, Barbosa JL (1999) Duchenne muscular dystrophy and concomitant metastatic alveolar rhabdomyosarcoma. J Pediatr Hematol Oncol 21: 528-530.

9. Leonela N Luce, Mercedes Abbate, Javier Cotignola, Florencia Giliberto (2017) Non-myogenic tumors display altered expression of dystrophin (DMD) and a high frequency of genetic alterations. Oncotarget 8: 145-155.

10. Segura LG, Lorenz JD, Weingarten TN, Scavonetto F, Bojanić K, et al. (2013) Anesthesia and Duchenne or Becker muscular dystrophy: Review of 117 anesthetic exposures. Paediatr Anaesth 23: 855-864.

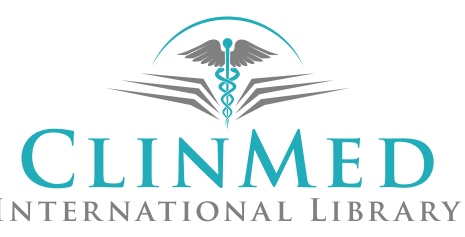

